\title{
Stabilization of Positive Switched Systems with Time-varying Delays under Asynchronous Switching
}

\author{
Mei Xiang, Zhengrong Xiang*, and Hamid Reza Karimi
}

\begin{abstract}
This paper investigates the state feedback stabilization problem for a class of positive switched systems with time-varying delays under asynchronous switching in the frameworks of continuous-time and discrete-time dynamics. The so-called asynchronous switching means that the switches between the candidate controllers and system modes are asynchronous. By constructing an appropriate co-positive type Lyapunov-Krasovskii functional and further allowing the functional to increase during the running time of active subsystems, sufficient conditions are provided to guarantee the exponential stability of the resulting closed-loop systems, and the corresponding controller gain matrices and admissible switching signals are presented. Finally, two illustrative examples are given to show the effectiveness of the proposed methods.
\end{abstract}

Keywords: Asynchronous switching, average dwell time, positive systems, switched systems, timevarying delays.

\section{INTRODUCTION}

Positive systems, whose states and outputs are positive (at least nonnegative) whenever the initial conditions and inputs are nonnegative, spread almost all over the areas. For instance, biomedicine [1], ecology [2], industrial engineering [3], TCP-like Internet congestion control [4], and so on. Very recently, positive switched systems, which contain a family of positive subsystems and a switching signal governing the switching among them, have been highlighted by many researchers due to their broad applications in communication systems [5], formation flying [6], and systems theory [7]. It should be pointed out that studying positive switched systems is more challenging because, in order to obtain some results, we have to combine the features of positive systems $[8,9]$ and switched systems [10-12].

In practice, time-delay phenomenon exists widely in engineering and social systems, for example, longdistance transportation systems, hydraulic pressure systems, networked control systems and so on. Timedelay often causes undesirable performance, even makes systems unstable. Therefore, many results have been

Manuscript received October 30, 2013; revised January 28, 2014; accepted February 15, 2014. Recommended by Associate Editor Izumi Masubuchi under the direction of Editor Yoshito Ohta.

This work was supported by the National Natural Science Foundation of China under Grant Nos. 60974027 and 61273120.

Mei Xiang and Zhengrong Xiang are with the School of Automation, Nanjing University of Science and Technology, Nanjing, 210094, P. R. China (e-mails: xiangmei1009@163.com, xiangzr@ mail.njust.edu.cn).

Hamid Reza Karimi is with the Department of Engineering, Faculty of Engineering and Science, University of Agder, N-4898 Grimstad, Norway (e-mails: hamid.r.karimi@uia.no).

* Corresponding author. reported for time-delay systems [13-15].

On the other hand, stability and stabilization problems of positive switched systems have been extensively studied in the past years, see for instance [16-25] and the references therein. It is worth pointing out that the aforementioned results are based on an assumption that the switching instants of the controllers coincide with those of system modes. However, as pointed out in [26,27], there inevitably exists asynchronous switching in actual operation, i.e., the switching instants of the controllers exceed or lag behind those of system modes. Thus, it is necessary to consider asynchronous switching for efficient control design. Some results on non-positive switched systems under asynchronous switching have been proposed in [26-33]. For positive switched systems, the asynchronously stabilization is more challenging because the designer concerns not only the stability of the system, but also the positive property of the system. However, to the best of the authors' knowledge, the asynchronous control problem for positive switched systems has not been fully investigated. Moreover, the asynchronous switching controller design method developed in the existing literature cannot be directly applied to positive switched systems, and this constitutes the main motivation of the present study.

In this paper, we are interested in studying the asynchronous stabilization problem for positive switched systems with time-varying delays. The main contribution of this paper is that: 1) by constructing a novel copositive type Lyapunov-Krasovskii functional which is allowed to increase during the running time of active subsystems with the mismatched controller, sufficient conditions for the existence of the stabilizing controllers for the underlying systems in both continuous-time and discrete-time contexts are developed; 2) The inequalities derived in this paper are decoupled and can be easily solved. 
The remainder of this paper is organized as follows. In Section 2, problem statements and necessary lemmas are given. In Section 3, based on the average dwell time approach, the stability and stabilization problems for both continuous-time and discrete-time positive switched systems with time-varying delays are developed. Two numerical examples are provided to show the effectiveness of the proposed approaches in Section 4. Concluding remarks are given in Section 5.

Notations: In this paper, $A \succ \succ 0(A \succeq \succeq 0)$ means that all elements of the matrix $A$ are positive (nonnegative). $A \succ \succ B(A \succeq \succeq B)$ means that $A-B \succ \succ 0(A-B \succeq \succeq 0)$. The notation $X \geq Y(X>Y)$ means that $X-Y$ is positive semi-definite (positive definite). $R^{n}$ is an $n$ dimensional real vector space and $R_{+}^{n}$ is a set of vectors whose elements are all positive; $R^{n \times s}$ is the set of all real matrices of $(n \times s)$-dimension. $A^{T}$ denotes the transpose of the matrix $A .\|x\|=\sum_{s=1}^{n}\left|x_{s}\right|$, where $x_{s}$ is the $s$-th element of $x \in R^{n}$. The set of all nonnegative integers is represented by $Z^{+}$.

\section{PROBLEM FORMULATION AND PRELIMINARIES}

Consider the following continuous-time switched linear system with time-varying delay:

$$
\left\{\begin{array}{l}
\dot{x}(t)=A_{\sigma(t)} x(t)+A_{d \sigma(t)} x(t-d(t))+B_{\sigma(t)} u(t), \\
x\left(t_{0}+\theta\right)=\varphi(\theta), \theta \in[-\tau, 0]
\end{array}\right.
$$

where $x(t) \in R^{n}$ and $u(t) \in R^{m}$ denote the system state and the control input, respectively; $\sigma(t):[0, \infty) \rightarrow \underline{N}=$ $\{1,2, \cdots, N\}$ is a piecewise constant function of time, called a switching signal; $N$ is the number of subsystems; Corresponding to the switching signal, we have the switching sequence $\Sigma=\left\{\left(t_{0}, \sigma\left(t_{0}\right)\right),\left(t_{1}, \sigma\left(t_{1}\right)\right), \cdots,\left(t_{q}, \sigma\left(t_{q}\right)\right)\right.$, $\cdots\}$, where $t_{0}$ is the initial instant and $t_{q}$ denotes the $q$-th switching instant; $A_{i}, A_{d i}$ and $B_{i}, i \in \underline{N}$, are known constant matrices with appropriate dimensions; $d(t)$ denotes the time-varying delay satisfying $0 \leq d(t) \leq \tau$ and $\dot{d}(t) \leq d<1$ for known constants $\tau$ and $d ; \varphi(\theta)$ is a continuous vector-valued initial function defined on the interval $[-\tau, 0]$.

Definition 1: System (1) is said to be a positive switched system if for any switching signals $\sigma(t)$ and any initial conditions $\varphi(\theta) \succeq \succeq 0, \theta \in[-\tau, 0], x(t) \succeq \succeq 0$ for all $t \geq t_{0}$.

Definition 2 [34]: $A$ is called a Metzler matrix, if the off-diagonal entries of the matrix $A$ are non-negative.

Definition 3 [35]: System (1) is said to be exponentially stable under switching signal $\sigma(t)$, if for initial conditions $x\left(t_{0}+\theta\right)=\varphi(\theta), \theta \in[-\tau, 0]$, there exist constants $\zeta>0$ and $\rho>0$ such that the solution of the system satisfies

$$
\|x(t)\| \leq \zeta\left\|x\left(t_{0}\right)\right\|_{C} e^{-\rho\left(t-t_{0}\right)}, \quad \forall t \geq t_{0},
$$

where $\left\|x\left(t_{0}\right)\right\|_{C}=\sup _{-\tau \leq \theta \leq 0}\left\|x\left(\theta+t_{0}\right)\right\|$.

Definition 4 [36]: For a switching signal $\sigma(t)$ and any $T_{2}>T_{1} \geq t_{0}$, let $N_{\sigma}\left(T_{1}, T_{2}\right)$ be the switching number of $\sigma(t)$ over the interval $\left[T_{1}, T_{2}\right)$. If $N_{\sigma}\left(T_{1}\right.$, $\left.T_{2}\right) \leq N_{0}+\left(T_{2}-T_{1}\right) / T_{a}$ holds for $T_{a}>0$ and $N_{0} \geq 0$, then $T_{a}$ is called the average dwell time and $N_{0}$ is called the chattering bound.

Remark 1: As stated in [36], $N_{0}=0$ implies that $\sigma(t)$ cannot switch at all on any interval of length smaller than $T_{a}$. In general, if we discard the first $N_{0}$ switches (more precisely, the smallest integer greater than $N_{0}$ ), the average time between consecutive switches is at least $T_{a}$. Without lose of generality, we choose $N_{0}=0$ in this paper.

A state feedback controller $u(t)=K_{\sigma(t)} x(t)$ for system (1) is considered, where $K_{i}(i \in \underline{N})$ are the controller gain matrices to be determined. However, due to the existence of asynchronous switching, the switching instants of the controller do not coincide exactly with those of the system mode. Without loss of generality, the asynchronous switching considered here means that the switching instants of controller lag behind those of the system mode. Then, the real control input will become

$$
u(t)=K_{\sigma\left(t-\Delta_{q}\right)} x(t), \quad \forall t \in\left[t_{q}, t_{q+1}\right),
$$

where $\Delta_{0}=0$, and $0 \leq \Delta_{q}<t_{q+1}-t_{q}(q=1,2, \ldots)$ represents the switching delay.

Remark 2: The delay time $0 \leq \Delta_{q}<t_{q+1}-t_{q}$ implies that there exists a period that the system mode and the controller operate synchronously during the interval $\left[t_{q}, t_{q+1}\right)$.

Denote $\sigma^{\prime}(t)$ as the real switching signal of the controller (4), that is, $\sigma^{\prime}(t)=\sigma\left(t-\Delta_{q}\right)$. Let the $i$-th subsystem be activated at the switching instant $t_{q}$ and the $j$-th subsystem be activated at the switching instant $t_{q+1}$, then the corresponding switching controllers are activated at the switching instants $t_{q}+\Delta_{q}$ and $t_{q+1}+\Delta_{q+1}$, respectively. Applying the controller (2) to system (1), the resulting closed-loop system is given by:

$$
\left\{\begin{array}{l}
\dot{x}(t)=\bar{A}_{i} x(t)+A_{d i} x(t-d(t)), t \in\left[t_{q}+\Delta_{q}, t_{q+1}\right) \\
\dot{x}(t)=\bar{A}_{i, j} x(t)+A_{d j} x(t-d(t)), t \in\left[t_{q+1}, t_{q+1}+\Delta_{q+1}\right),
\end{array}\right.
$$

where $\bar{A}_{i}=A_{i}+B_{i} K_{i}$ and $\bar{A}_{i, j}=A_{j}+B_{j} K_{i}$.

Lemma 1: System (3) is positive if and only if $\bar{A}_{i}$ and $\bar{A}_{i, j}$ are Metzler matrices, and $A_{d i} \succeq \succeq 0, \forall i, j \in \underline{N}$, $i \neq j$.

Proof: This Lemma can be directly obtained from Proposition 1 in [16].

Furthermore, we consider the following discrete-time switched system with time-varying delay:

$$
\left\{\begin{array}{l}
x(k+1)=A_{\sigma(k)} x(k)+A_{d \sigma(k)} x(k-d(k))+B_{\sigma(k)} u(k), \\
x\left(k+k_{0}\right)=\varphi(k), k=-\bar{d},-\bar{d}+1, \cdots,-1,0,
\end{array}\right.
$$


where $x(k) \in R^{n}$ and $u(k) \in R^{m}$ denote the system state and the control input, respectively; $\sigma(k): Z^{+} \rightarrow \underline{N}=$ $\{1,2, \cdots, N\} \quad$ is the switching signal; $\sigma(k)=i \in \underline{N}$ means that the i-th subsystem is active; $N$ is the number of subsystems; Corresponding to the switching signal, we have the switching sequence $\bar{\Sigma}=\left\{\left(k_{0}, \sigma\left(k_{0}\right)\right),\left(k_{1}\right.\right.$, $\left.\left.\sigma\left(k_{1}\right)\right), \cdots,\left(k_{l}, \sigma\left(k_{l}\right)\right), \cdots\right\}$, where $k_{0}=0$ is the initial instant and $k_{l}$ denotes the 1-th switching instant; $A_{i}, A_{d i}$ and $B_{i}, i \in N$, are constant matrices with appropriate dimensions; $d(k)$ denotes the time-varying delay satisfying $0<\underline{d} \leq d(k) \leq \bar{d}$ for known constants $\underline{d}$ and $\bar{d} ; \varphi(k)$ is a discrete vector-valued initial function.

Definition 5: System (4) is said to be positive if, for any initial conditions $\varphi(k) \succeq \succeq 0, k=-\bar{d},-\bar{d}+1, \cdots,-1,0$, and any switching signals $\sigma(k), x(k) \succeq \succeq 0$ holds for all $k \geq k_{0}$.

Definition 6 [36]: System (4) is said to be exponentially stable under switching signal $\sigma(k)$ if for initial conditions $x\left(k+k_{0}\right)=\varphi(k), k=-\bar{d},-\bar{d}+1, \cdots,-1,0$, ther e exist constants $\varepsilon>0$ and $\xi>0$ such that $\|x(k)\| \leq$ $\varepsilon e^{-\xi\left(k-k_{0}\right)}\left\|x\left(k_{0}\right)\right\|_{C}, \forall k \geq k_{0}$, where $\left\|x\left(k_{0}\right)\right\|_{C}=$ sup $\left\|x\left(k_{0}+\theta\right)\right\|$.

Definition 7 [37]: For any $k_{2}>k_{1} \geq k_{0}$, let $N_{\sigma}\left(k_{1}\right.$, $k_{2}$ ) be the switching number of $\sigma(k)$ over the interval $\left[k_{1}, k_{2}\right)$. If $N_{\sigma}\left(k_{1}, k_{2}\right) \leq N_{0}+\left(k_{2}-k_{1}\right) / \tau_{a}$ holds for $\tau_{a}>0$ and $N_{0} \geq 0$, then $\tau_{a}$ is called the average dwell time and $N_{0}$ is called the chattering bound.

The asynchronous state feedback controller for system (4) can be written as

$$
u(k)=K_{\sigma\left(k-\Delta_{l}\right)} x(k), \quad \forall k \in\left[k_{l}, k_{l+1}\right),
$$

where $\Delta_{0}=0$ and $0 \leq \Delta_{l}<k_{l+1}-k_{l}(l=1,2, \ldots)$ represents the switching delay.

Denote $\sigma^{\prime}(k)$ as the real switching signal of the controller (5), then $\sigma^{\prime}(k)=\sigma\left(k-\Delta_{l}\right)$. Letting $\sigma\left(k_{l}\right)=i$ and $\sigma\left(k_{l+1}\right)=j$, we have $\sigma\left(k_{l}+\Delta_{l}\right)=i, \sigma\left(k_{l+1}+\Delta_{l+1}\right)$ $=j$, and the resulting closed-loop system is given by

$$
\left\{\begin{array}{l}
x(k+1)=\bar{A}_{i} x(k)+A_{d i} x(k-d(k)), k \in\left[k_{l}+\Delta_{l}, k_{l+1}\right) \\
x(k+1)=\bar{A}_{i, j} x(k)+A_{d j} x(k-d(k)), k \in\left[k_{l+1}, k_{l+1}+\Delta_{l+1}\right),
\end{array}\right.
$$

where $\bar{A}_{i}=A_{i}+B_{i} K_{i}$ and $\bar{A}_{i, j}=A_{j}+B_{j} K_{i}$. [38].

The following lemma is an extension of Lemma 3 in

Lemma 2: System (6) is positive if and only if $\bar{A}_{i} \succeq \succeq 0, \bar{A}_{i, j} \succeq \succeq 0$ and $A_{d i} \succeq \succeq 0, \forall i, j \in \underline{N}, i \neq j$.

\section{MAIN RESULTS}

\subsection{Continuous-time case}

Before giving the main results, we first present the following lemmas which will be essential for our later development.
Lemma 3: Consider the following positive system

$$
\left\{\begin{array}{l}
\dot{x}(t)=A x(t)+A_{d} x(t-d(t)), \\
x\left(t_{0}+\theta\right)=\varphi(\theta), \theta \in[-\tau, 0],
\end{array}\right.
$$

where $A$ is a Metzler constant matrix and $A_{d} \succeq \succeq 0$ is a constant matrix; $d(t)$ denotes the time-varying delay satisfying $0 \leq d(t) \leq \tau$ and $\dot{d}(t) \leq d<1 ; \varphi(\theta) \succ \succ 0, \theta \in$ $[-\tau, 0]$. For a given positive scalar $\alpha$, if there exist vectors $v, v \in R_{+}^{n}$, such that

$$
\begin{aligned}
& A^{T} v+\alpha v+v \preceq \preceq 0, \\
& A_{d}^{T} v-(1-d) e^{-\alpha \tau} v \preceq \preceq 0,
\end{aligned}
$$

then along the trajectory of system (7), we have

$$
V(t) \leq e^{-\alpha\left(t-t_{0}\right)} V\left(t_{0}\right) .
$$

Proof: Applying Lemma 2 in [39,40], and letting $\varsigma=0$ and $\vartheta=0$, the lemma can be directly obtained.

Lemma 4: Consider system (7), for a given positive scalar $\beta$, if there exist vectors $v, v \in R_{+}^{n}$, such that

$$
\begin{aligned}
& A^{T} v-\beta v+v \preceq \preceq 0, \\
& A_{d}^{T} v-(1-d) v \preceq \preceq 0,
\end{aligned}
$$

then along the trajectory of system (7), we have

$$
V(t) \leq e^{\beta\left(t-t_{0}\right)} V\left(t_{0}\right) .
$$

Proof: Applying Lemma 3 in [39], and letting $\varsigma=0$ and $\vartheta=0$, this lemma can be directly obtained.

Remark 3: Lemmas 3 and 4 provide the decay estimation and growth estimation of the co-positive type Lyapunov-Krasovskii functional, respectively. They will be used for the asynchronous controller design of positive switched system (1).

The following theorem presents a sufficient condition for the existence of an asynchronous stabilizing controller for positive switched system (1).

Theorem 1: Consider positive switched system (1). For given positive scalars $\alpha, \beta, \lambda_{i, j}$, and vectors $\tilde{v}_{i} \in$ $R_{+}^{m}$, if there exist vectors $v_{i}, v_{i}, v_{i, j}, v_{i, j} \in R_{+}^{n}$, and $h_{i} \in R^{n}$, such that, $\forall(i, j) \in \underline{N} \times \underline{N}, i \neq j$,

$$
\begin{aligned}
& A_{i}^{T} v_{i}+\alpha v_{i}+h_{i}+v_{i} \preceq \preceq 0, \\
& A_{d i}^{T} v_{i}-(1-d) e^{-\alpha \tau} v_{i} \preceq \preceq 0, \\
& \lambda_{i, j} \tilde{v}_{i}^{T} B_{i}^{T} v_{i} \leq \tilde{v}_{i}^{T} B_{j}^{T} v_{i, j}, \quad h_{i} \preceq \preceq 0, \\
& \tilde{v}_{i}^{T} B_{i}^{T} v_{i}>0, \\
& A_{j}^{T} v_{i, j}-\beta v_{i, j}+\lambda_{i, j} h_{i}+v_{i, j} \preceq \preceq 0, \\
& A_{d j}^{T} v_{i, j}-(1-d) v_{i, j} \preceq \preceq 0, \\
& \mathbf{I}_{g}^{T}\left(\tilde{v}_{i}^{T} B_{i}^{T} v_{i} A_{i}^{T}+h_{i} \tilde{v}_{i}^{T} B_{i}^{T}\right) \mathbf{I}_{l} \geq 0, \quad g \neq l, \\
& \mathbf{I}_{g}^{T}\left(\tilde{v}_{i}^{T} B_{i}^{T} v_{i} A_{j}^{T}+h_{i} \tilde{v}_{i}^{T} B_{j}^{T}\right) \mathbf{I}_{l} \geq 0, \quad g \neq l,
\end{aligned}
$$

where $\mathbf{I}_{g}=[\overbrace{0 \ldots 0}^{g-1} \overbrace{0 \ldots 0}^{n-g}]$ and $\mathbf{I}_{l}=[\overbrace{0 \ldots 0}^{l-1} \overbrace{0 \ldots 0}^{n-l}]$, then 
under the controller (2) with

$$
K_{i}=\frac{1}{\tilde{v}_{i}^{T} B_{i}^{T} v_{i}} \tilde{v}_{i} h_{i}^{T},
$$

the closed-loop system (3) is exponentially stable for any switching signal $\sigma(t)$ with average dwell time satisfying

$$
T_{a}>T_{a}^{*}=\left(\Delta(\alpha+\beta)+\ln \left(\mu_{1} \mu_{2} \mu\right)\right) / \alpha,
$$

where $\Delta=\max \left\{\Delta_{q}\right\}, q=1,2, \ldots, \mu=e^{\tau(\alpha+\beta)}, \mu_{1}$ and $\mu_{2}$ satisfy

$$
\begin{array}{ll}
v_{j} \preceq \preceq \mu_{1} v_{i, j}, & v_{j} \preceq \preceq \mu_{1} v_{i, j}, \quad v_{i, j} \preceq \preceq \mu_{2} \mu v_{i}, \\
v_{i, j} \preceq \preceq \mu_{2} v_{i}, & \forall(i, j) \in \underline{N} \times \underline{N}, \quad i \neq j .
\end{array}
$$

Proof: When $t \in\left[t_{q}+\Delta_{q}, t_{q+1}\right)$, the closed-loop system (3) can be written as

$$
\dot{x}(t)=\bar{A}_{i} x(t)+A_{d i} x(t-d(t)) .
$$

Equation (20) implies that $\tilde{v}_{i}^{T} B_{i}^{T} v_{i} A_{i}^{T}+h_{i} \tilde{v}_{i}^{T} B_{i}^{T}$ is a Metzler matrix. Then it follows from (17) that

$$
A_{i}^{T}+\frac{1}{\tilde{v}_{i}^{T} B_{i}^{T} v_{i}} h_{i} \tilde{v}_{i}^{T} B_{i}^{T}
$$

is a Metzler matrix. From (22), one has $\bar{A}_{i}=A_{i}+B_{i} K_{i}$ is a Metzler matrix.

Consider the following co-positive type LyapunovKrasovskii functional for system (25)

$$
V_{i}(t)=x^{T}(t) v_{i}+\int_{t-d(t)}^{t} e^{\alpha(-t+s)} x^{T}(s) v_{i} d s .
$$

Using (22), we have $h_{i}=K_{i}^{T} B_{i}^{T} v_{i}$, then according to Lemma 3, it can be obtained from (14)-(15) that, for $t \in\left[t_{q}+\Delta_{q}, t_{q+1}\right)$,

$$
V_{i}(t) \leq e^{-\alpha\left(t-t_{q}-\Delta_{q}\right)} V_{i}\left(t_{q}+\Delta_{q}\right) .
$$

When $t \in\left[t_{q+1}, t_{q+1}+\Delta_{q+1}\right)$, the closed-loop system can be written as

$$
\dot{x}(t)=\bar{A}_{i, j} x(t)+A_{d j} x(t-d(t)) .
$$

Similarly, one can obtain from (16), (17), and (21) that $\bar{A}_{i, j}=A_{j}+B_{j} K_{i}$ is a Metzler matrix.

Choose a co-positive type Lyapunov-Krasovskii functional for system (28) as follows:

$$
V_{i, j}(x(t))=x^{T}(t) v_{i, j}+\int_{t-d(t)}^{t} e^{\beta(t-s)} x^{T}(s) v_{i, j} d s .
$$

From (22), it is easy to get that

$$
K_{j}^{T} B_{j}^{T} v_{i, j}=h_{i} \frac{\tilde{v}_{i}^{T} B_{j}^{T} v_{i, j}}{\tilde{v}_{i}^{T} B_{j}^{T} v_{i}} .
$$

It follows from (16)-(18) that

$$
A_{j}^{T} v_{i, j}-\beta v_{i, j}+K_{j}^{T} B_{j}^{T} v_{i, j}+v_{i, j} \preceq \preceq 0 .
$$

Using (19), (30), and applying Lemma 4, one obtains

$$
V_{i, j}(t) \leq e^{\beta\left(t-t_{q+1}\right)} V_{i, j}\left(t_{q+1}\right) .
$$

Denote $t_{1}, \cdots, t_{f}$ as the switching instants on the interval $\left[t_{0}, t\right)$, and consider the following LyapunovKrasovskii functional candidate for system (3): When $t \in\left[t_{\kappa}+\Delta_{\kappa}, t_{\kappa+1}\right), \kappa=0,1,2, \cdots, f$,

$$
V_{\sigma(t)}(t)=x^{T}(t) v_{\sigma(t)}+\int_{t-d(t)}^{t} e^{\alpha(-t+s)} x^{T}(s) v_{\sigma(t)} d s .
$$

When $t \in\left[t_{\kappa}, t_{\kappa}+\Delta_{\kappa}\right), \kappa=1,2, \cdots, f$,

$$
\begin{aligned}
V_{\sigma^{\prime}(t), \sigma(t)}(t)= & x^{T}(t) v_{\sigma^{\prime}(t), \sigma(t)} \\
& +\int_{t-d(t)}^{t} e^{\beta(t-s)} x^{T}(s) v_{\sigma^{\prime}(t), \sigma(t)} d s .
\end{aligned}
$$

From (24), (27) and (31), for $t \in\left[t_{f}+\Delta_{f}, t_{f+1}\right)$, we obtain that

$$
\begin{aligned}
V_{\sigma(t)}(t) \leq & e^{-\alpha\left(t-t_{f}-\Delta_{f}\right)} V_{\sigma\left(t_{f}+\Delta_{f}\right)}\left(t_{f}+\Delta_{f}\right) \\
\leq & \mu_{1} e^{-\alpha\left(t-t_{f}-\Delta_{f}\right)} e^{\beta \Delta_{f}} V_{\sigma^{\prime}\left(t_{f}\right), \sigma\left(t_{f}\right)}\left(t_{f}\right) \\
\leq & \mu_{1} \mu_{2} \mu e^{-\alpha\left(t-t_{f}-\Delta_{f}\right)} e^{\beta \Delta_{f}} V_{\sigma\left(t_{f}\right)}\left(t_{f}^{-}\right) \\
\leq & \mu_{1} \mu_{2} \mu e^{-\alpha\left(t-t_{f}-\Delta\right)} e^{\beta \Delta} V_{\sigma\left(t_{f}^{-}\right)}\left(t_{f}^{-}\right) \\
\leq & \cdots \\
\leq & \left(\mu_{1} \mu_{2} \mu\right)^{\left(t-t_{0}\right) / T_{a}} e^{-\alpha\left(t-t_{0}\right)} e^{\Delta(\alpha+\beta)\left(t-t_{0}\right) / T_{a}} \\
& \times V_{\sigma\left(t_{0}\right)}\left(t_{0}\right) .
\end{aligned}
$$

From (23) and (32), one has

$$
V_{\sigma(t)}(t) \leq e^{-\rho\left(t-t_{0}\right)} V_{\sigma\left(t_{0}\right)}\left(t_{0}\right),
$$

where $\rho=-\left(\Delta(\alpha+\beta)+\ln \left(\mu_{1} \mu_{2} \mu\right)\right) / T_{a}+\alpha>0$.

Thus there exists a constant $\zeta>0$ such that the system state satisfies

$$
\|x(t)\| \leq \zeta e^{-\rho\left(t-t_{0}\right)}\left\|x\left(t_{0}\right)\right\|_{C} .
$$

The proof is completed.

Remark 4: From Theorem 1, it can be seen that a smaller $\alpha$ and a larger $\beta$ will be favorable to the feasibility of inequalities (14)-(21). In view of this, we put forward the following procedure for determining the controller gain matrices $K_{i}$ and the desired $T_{a}^{*}$.

\section{Algorithm 1}

Step 1: Input the system matrices $A_{i}, A_{d i}$ and $B_{i}$, and given $\lambda_{i, j}>0$ and vectors $\tilde{v}_{i} \in R_{+}^{m}$.

Step 2: Take $\alpha$ and $\beta$ (For the first time, we can choose a larger $\alpha>0$ and a smaller $\beta>0$ ), and solve (14)-(21).

Step 3: If there is a feasible solution, go to Step 5.

Step 4: If there inequalities are infeasible, decrease $\alpha$ and increase $\beta$ appropriately, and go to Step 2 .

Step 5: Compute the controller gain matrices $K_{i}$ by (22), and calculate $\mu_{1}$ and $\mu_{2}$ by the following optimization approach

minimize $\mu_{1}+\mu_{2}$ s.t. (24). 
Step 6: Calculate $T_{a}^{*}$ by (23).

When $\Delta=0$, i.e., the controllers are switched synchronously with the subsystems, we can get the following result.

Corollary 1: Consider positive switched system (1). For given positive scalars $\alpha, \beta$, and vectors $\tilde{v}_{i} \in R_{+}^{m}$, if there exist vectors $v_{i}, v_{i} \in R_{+}^{n}$ and $h_{i} \in R^{n}$, such that (14), (15), (17), and (20) hold, then under the state feedback controller $u(t)=K_{\sigma(t)} x(t)$ with (22), the resulting closed-loop system (3) is exponentially stable for any switching signal $\sigma(t)$ with average dwell time satisfying $T_{a}>T_{a}^{*}=\ln \mu_{1} / \alpha$, where $\mu_{1} \geq 1$ satisfies $v_{i} \preceq \preceq \mu_{1} v_{j}, v_{i} \preceq \preceq \mu_{1} v_{j}, \forall(i, j) \in \underline{N} \times \underline{N}, i \neq j$.

In what follows, we will give the asynchronous switching controller design scheme for discrete-time positive switched system (4).

\subsection{Discrete-time case}

Before giving the result, we first present the following lemma.

Lemma 5: Consider the following positive system

$$
\left\{\begin{array}{l}
x(k+1)=A x(k)+A_{d} x(k-d(k)), \\
x\left(k+k_{0}\right)=\varphi(k), \quad k=-\bar{d},-\bar{d}+1, \cdots,-1,0,
\end{array}\right.
$$

where $A \succeq \succeq 0$ and $A_{d} \succeq \succeq 0$ are constant matrices with appropriate dimensions; $d(k)$ denotes the time-varying delay satisfying $0<\underline{d} \leq d(k) \leq \bar{d} ; \quad \varphi(k) \succeq \succeq 0, \quad k=-\bar{d}$, $-\bar{d}+1, \cdots,-1,0$. For a given positive scalar $\eta$, if there exist vectors $v, v \in R_{+}^{n}$, such that

$$
\begin{aligned}
& A^{T} v-e^{-\eta} v+(\bar{d}-\underline{d}+1) v \preceq \preceq 0, \\
& A_{d}^{T} v-e^{-\eta \bar{d}} v \preceq \preceq 0,
\end{aligned}
$$

then along the trajectory of system (34), we have

$$
V(k) \leq e^{-\eta\left(k-k_{0}\right)} V\left(k_{0}\right) .
$$

Proof: Consider the following co-positive type Lyapunov-Krasovskii functional:

$$
\begin{aligned}
V(k)= & x^{T}(k) v+\sum_{s=k-d(k)}^{k-1} e^{\eta(-k+s+1)} x^{T}(s) v \\
& +\sum_{\theta=-\bar{d}+1}^{-d} \sum_{s=k+\theta}^{k-1} e^{\eta(-k+s+1)} x^{T}(s) v .
\end{aligned}
$$

Along the trajectory of (34), we have

$$
\begin{aligned}
& V(k+1)-e^{-\eta} V(k) \leq x^{T}(k)\left(A^{T} v-e^{-\eta} v+(\bar{d}-\underline{d}+1) v\right) \\
& \quad+x^{T}(k-d(k))\left(A_{d}^{T} v-e^{-\eta \bar{d}} v\right) .
\end{aligned}
$$

It follows from (35)-(36) that

$$
V(k) \leq e^{-\eta\left(k-k_{0}\right)} V\left(k_{0}\right) .
$$

The proof is completed.

The following theorem presents a sufficient condition for the existence of an asynchronous stabilizing controller for system (4).
Theorem 2: Consider positive switched system (4). For given positive scalars $\eta, \gamma, \lambda_{i, j}$, and vectors $\tilde{v}_{i} \in$ $R_{+}^{m}$, if there exist vectors $v_{i}, v_{i}, v_{i, j}, v_{i, j} \in R_{+}^{n}$, and $h_{i} \in R^{n}$, such that, $\forall(i, j) \in \underline{N} \times \underline{N}, i \neq j$,

$$
\begin{aligned}
& A_{i}^{T} v_{i}+h_{i}-e^{-\eta} v_{i}+(\bar{d}-\underline{d}+1) v_{i} \preceq \preceq, \\
& A_{d i}^{T} v_{i}-e^{-\eta \bar{d}} v_{i} \preceq \preceq 0, \\
& \lambda_{i, j} \tilde{v}_{i}^{T} B_{i}^{T} v_{i} \leq \tilde{v}_{i}^{T} B_{j}^{T} v_{i, j}, \quad h_{i} \preceq \preceq 0, \\
& \tilde{v}_{i}^{T} B_{i}^{T} v_{i}>0, \\
& A_{j}^{T} v_{i, j}-e^{\gamma} v_{i, j}+\lambda_{i, j} h_{i}+(\bar{d}-\underline{d}+1) v_{i, j} \preceq \preceq 0, \\
& A_{d j}^{T} v_{i, j}-e^{\gamma} \underline{d} v_{i, j} \preceq \preceq 0, \\
& \tilde{v}_{i}^{T} B_{i}^{T} v_{i} A_{i}^{T}+h_{i} \tilde{v}_{i}^{T} B_{i}^{T} \succeq \succeq 0, \\
& \tilde{v}_{i}^{T} B_{i}^{T} v_{i} A_{j}^{T}+h_{i} \tilde{v}_{i}^{T} B_{j}^{T} \succeq \succeq 0,
\end{aligned}
$$

then under the controller (5) with

$$
K_{i}=\frac{1}{\tilde{v}_{i}^{T} B_{i}^{T} v_{i}} \tilde{v}_{i} h_{i}^{T},
$$

the closed-loop system (6) is exponentially stable for any switching signal $\sigma(t)$ with average dwell time satisfying

$$
\tau_{a}>\tau_{a}^{*}=\left(\Delta^{\prime}(\eta+\gamma)+\ln \left(\mu_{1} \mu_{2} \mu\right)\right) / \eta,
$$

where $\Delta^{\prime}=\max \left\{\Delta_{l}\right\}, l=1,2, \ldots, \mu_{1}, \mu_{2}$ and $\mu$ satisfy

$$
\begin{aligned}
& \mu=e^{\bar{d}(\eta+\gamma)}, \quad v_{j} \preceq \preceq \mu_{1} v_{i, j}, \quad v_{j} \preceq \preceq \mu_{1} v_{i, j}, \\
& v_{i, j} \preceq \preceq \mu_{2} \mu v_{i}, \quad v_{i, j} \preceq \preceq \mu_{2} v_{i}, \\
& \forall(i, j) \in \underline{N} \times \underline{N}, \quad i \neq j .
\end{aligned}
$$

Proof: Similarly, one can obtain from (41), (44)-(46) that $A_{i}+B_{i} K_{i} \succeq \succeq 0$ and $A_{j}+B_{j} K_{i} \succeq \succeq 0$.

Denote $k_{1}, \cdots, k_{w}$ the switching instants during the interval $\left[k_{0}, k\right)$, and consider the following LyapunovKrasovskii functional candidates for system (6): When $k \in\left[k_{z}+\Delta_{z}, k_{z+1}\right), z=0,1,2, \cdots, w$,

$$
\begin{aligned}
V_{\sigma(k)}(k)= & x^{T}(k) v_{\sigma(k)}+\sum_{s=k-d(k)}^{k-1} e^{\eta(-k+s+1)} x^{T}(s) v_{\sigma(k)} \\
& +\sum_{\theta=-\bar{d}+1}^{-d} \sum_{s=k+\theta}^{k-1} e^{\eta(-k+s+1)} x^{T}(s) v_{\sigma(k)} .
\end{aligned}
$$

When $k \in\left[k_{z}, k_{z}+\Delta_{z}\right), z=1,2, \cdots, w$,

$$
\begin{aligned}
V_{\sigma^{\prime}(k), \sigma(k)}(k)= & x^{T}(k) v_{\sigma^{\prime}(k), \sigma(k)} \\
& +\sum_{s=k-d(k)}^{k-1} e^{\gamma(k-s-1)} x^{T}(s) v_{\sigma^{\prime}(k), \sigma(k)} \\
& +\sum_{\theta=-\bar{d}+1}^{-d} \sum_{s=k+\theta}^{k-1} e^{\gamma(k-s-1)} x^{T}(s) v_{\sigma^{\prime}(k), \sigma(k)} .
\end{aligned}
$$

Then similar to the proof of Theorem 1, we can obtain from (38)-(48) and Lemma 5 that

$$
V_{\sigma(k)}(k) \leq e^{-\xi\left(k-k_{0}\right)} V_{\sigma\left(k_{0}\right)}\left(k_{0}\right),
$$


where $\xi=\eta-\left(\ln \left(\mu_{1} \mu_{2} \mu\right)+\Delta^{\prime}(\eta+\gamma)\right) / \tau_{a}>0$.

It follows that there exists a constant $\varepsilon>0$ such that the system state satisfies

$$
\|x(k)\| \leq \varepsilon e^{-\xi\left(k-k_{0}\right)}\left\|x\left(k_{0}\right)\right\|_{C} .
$$

The proof is completed.

When $\Delta=0$, we can get the following result.

Corollary 2: Consider positive switched system (4). For given positive scalars $\eta, \gamma$, and vectors $\tilde{v}_{i} \in R_{+}^{m}$, if there exist vectors $v_{i}, v_{i} \in R_{+}^{n}$ and $h_{i} \in R^{n}$, such that (38), (39), (41), and (44) hold, then under the state feedback controller $u(k)=K_{\sigma(k)} x(k)$ with (46), the closed-loop system (6) is exponentially stable for any switching signal $\sigma(k)$ with average dwell time satisfying $\tau_{a}>\tau_{a}^{*}=\ln \mu_{1} / \eta$, where $\mu_{1} \geq 1$ satisfies $v_{i} \preceq \preceq \mu_{1} v_{j}$, $v_{i} \preceq \preceq \mu_{1} v_{j}, \forall i, j \in \underline{N}, i \neq j$.

Based on Theorem 2, we give the following procedure to obtain the controller gain matrices $K_{i}$ and the desired $\tau_{a}^{*}$.

\section{Algorithm 2}

Step 1: Input the system matrices $A_{i}, A_{d i}$ and $B_{i}$, and given $\lambda_{i, j}>0$ and vectors $\tilde{v}_{i} \in R_{+}^{m}$.

Step 2: Take $\eta$ and $\gamma$ (For the first time, we can choose a larger $\eta>0$ and a smaller $\gamma>0$ ), and solve (38)-(45).

Step 3: If there is a feasible solution, and go to Step 2; otherwise, go to Step 5.

Step 4: If there inequalities are infeasible, decrease $\eta$ and increase $\gamma$ appropriately, and go to Step 2 .

Step 5: Compute the controller gain matrices $K_{i}$ by (46), and calculate $\mu_{1}$ and $\mu_{2}$ by the following optimization approach

$$
\begin{gathered}
\text { minimize } \mu_{1}+\mu_{2} \\
\text { s.t. }(48) .
\end{gathered}
$$

Step 6: Calculate $\tau_{a}^{*}$ by (47).

\section{NUMERICAL EXAMPLES}

In this section, two numerical examples will be presented to demonstrate the validity of our developed theoretical results.

Example 1: Consider system (1) with the following parameters

$$
\begin{aligned}
& A_{1}=\left[\begin{array}{cc}
-3.5 & 3 \\
5.8 & -4.8
\end{array}\right], \quad A_{d 1}=\left[\begin{array}{cc}
0 & 0 \\
0.2 & 0.18
\end{array}\right], \quad B_{1}=\left[\begin{array}{l}
0.5 \\
0.2
\end{array}\right], \\
& A_{2}=\left[\begin{array}{cc}
-3 & 3.3 \\
4.3 & -5
\end{array}\right], \quad A_{d 2}=\left[\begin{array}{cc}
0.2 & 0 \\
0.1 & 0.2
\end{array}\right], \quad B_{2}=\left[\begin{array}{l}
0.6 \\
0.3
\end{array}\right], \\
& \tau=0.1, \quad d=0.1, \quad \Delta=0.3 .
\end{aligned}
$$

Take $\alpha=1.8, \beta=1.5, \tilde{v}_{1}=0.4, \tilde{v}_{2}=0.6, \lambda_{1,2}=0.4$ and $\lambda_{2,1}=1$, then solving (14)-(21) in Theorem 1 gives rise to

$$
v_{1}=\left[\begin{array}{l}
18.5311 \\
13.1778
\end{array}\right], \quad v_{2}=\left[\begin{array}{l}
10.1169 \\
11.0389
\end{array}\right], \quad v_{1}=\left[\begin{array}{l}
16.3290 \\
13.7281
\end{array}\right],
$$

$$
\begin{aligned}
& v_{2}=\left[\begin{array}{l}
15.2088 \\
14.5994
\end{array}\right], \quad v_{1,2}=\left[\begin{array}{l}
20.1997 \\
15.6530
\end{array}\right], \quad v_{2,1}=\left[\begin{array}{l}
19.3433 \\
16.2392
\end{array}\right], \\
& v_{2,1}=\left[\begin{array}{l}
28.6204 \\
29.3711
\end{array}\right], \quad v_{1,2}=\left[\begin{array}{l}
27.0522 \\
25.2969
\end{array}\right], \\
& h_{1}=\left[\begin{array}{l}
-71.6062 \\
-38.0025
\end{array}\right], \quad h_{2}=\left[\begin{array}{l}
-59.5055 \\
-21.6463
\end{array}\right] .
\end{aligned}
$$

By (22), the gain matrices $K_{i}, i=1,2$, can be obtained

$$
K_{1}=\left[\begin{array}{ll}
-6.0167 & -3.1932
\end{array}\right], K_{2}=\left[\begin{array}{ll}
-6.3426 & -2.3073
\end{array}\right] \text {. }
$$

Furthermore, according to (24), we have $\mu_{1}=0.9580$, $\mu_{2}=2.9698$ and $\mu=1.3910$. From (23), it can be obtained that $T_{a}^{*}=1.0979$. Choosing $T_{a}=1.5$, the simulation results are shown in Figs. 1-2, where the initial conditions are $x(0)=\left[\begin{array}{ll}0.2 & 0.1\end{array}\right]^{T}$ and $x(\theta)=$ $\left[\begin{array}{ll}0 & 0\end{array}\right]^{T}, \theta \in[-0.1,0)$.

From Figs. 1-2, we can see that the resulting closedloop system is positive and exponentially stable, which indicates that the proposed method is effective.

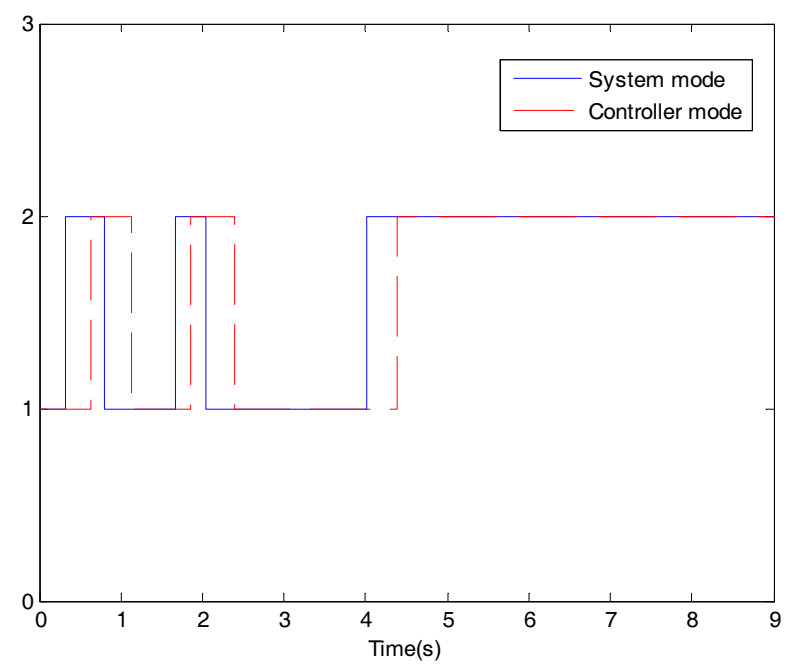

Fig. 1. Switching signal in Example 1.

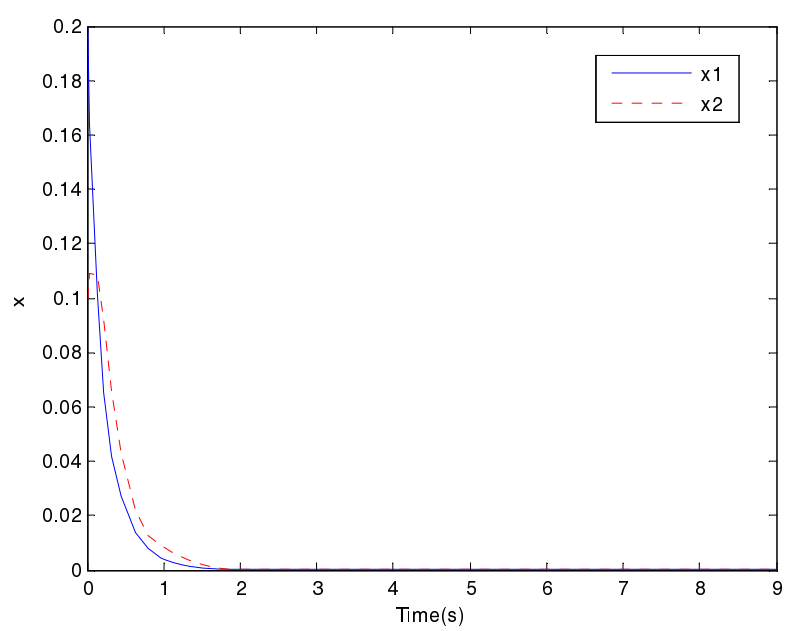

Fig. 2. State response of the closed-loop system in Example 1. 
Example 2: Consider system (4) with the following parameters

$$
\begin{aligned}
& A_{1}=\left[\begin{array}{cc}
0.6 & 0.48 \\
0.6 & 0.5
\end{array}\right], \quad A_{d 1}=\left[\begin{array}{cc}
0.1 & 0 \\
0 & 0
\end{array}\right], \quad B_{1}=\left[\begin{array}{l}
0.3 \\
0.3
\end{array}\right], \\
& A_{2}=\left[\begin{array}{cc}
0.5 & 0.45 \\
0.62 & 0.54
\end{array}\right], \quad A_{d 2}=\left[\begin{array}{cc}
0.1 & 0 \\
0 & 0
\end{array}\right], \quad B_{2}=\left[\begin{array}{l}
0.25 \\
0.32
\end{array}\right], \\
& \underline{d}=1, \quad \bar{d}=2, \quad \Delta^{\prime}=1 .
\end{aligned}
$$

Take $\eta=0.32, \gamma=0.02, \tilde{v}_{1}=1, \tilde{v}_{2}=1, \lambda_{1,2}=1.2$ and $\lambda_{2,1}=1.2$, then solving (38)-(45) in Theorem 2 gives rise to

$$
\begin{aligned}
& v_{1}=\left[\begin{array}{l}
14.5190 \\
15.2754
\end{array}\right], \quad v_{2}=\left[\begin{array}{l}
15.0434 \\
16.6506
\end{array}\right], \quad v_{1}=\left[\begin{array}{l}
2.9007 \\
1.7853
\end{array}\right], \\
& v_{2}=\left[\begin{array}{l}
3.0949 \\
1.8310
\end{array}\right], \quad v_{2,1}=\left[\begin{array}{l}
20.8460 \\
20.6063
\end{array}\right], \quad v_{1,2}=\left[\begin{array}{l}
20.0610 \\
22.5155
\end{array}\right], \\
& v_{2,1}=\left[\begin{array}{l}
4.8236 \\
3.8481
\end{array}\right], \quad v_{1,2}=\left[\begin{array}{l}
4.8193 \\
4.0905
\end{array}\right],
\end{aligned}
$$

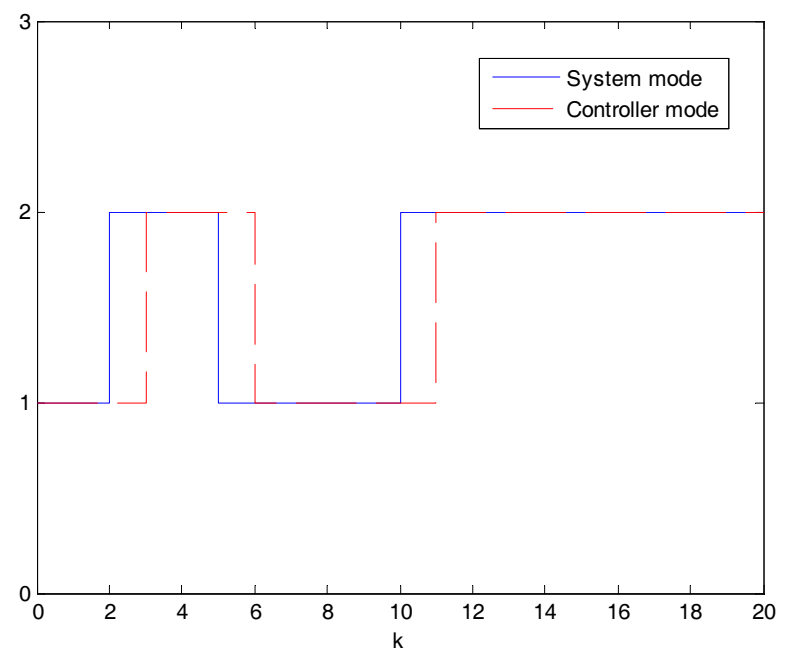

Fig. 3. Switching signal in Example 2.

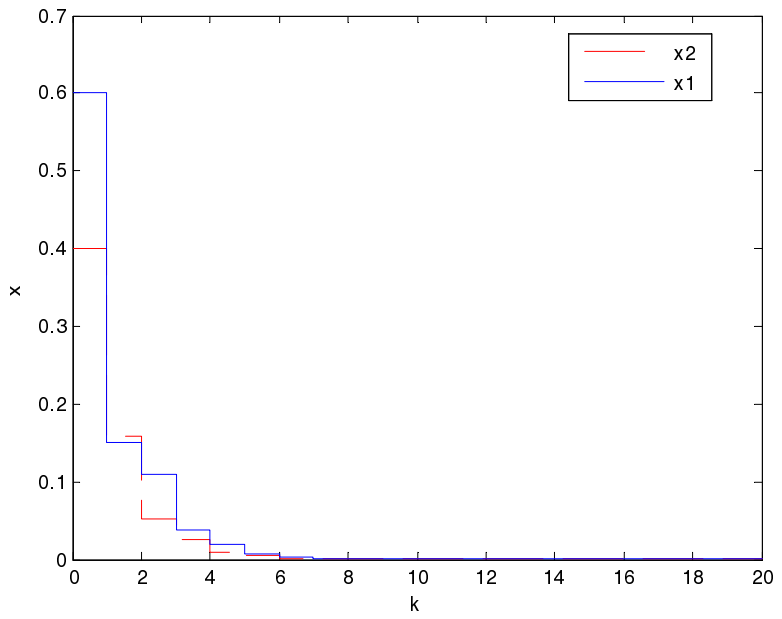

Fig. 4. State response of the closed-loop system in Example 2.

$$
h_{1}=\left[\begin{array}{c}
-14.0503 \\
-8.7643
\end{array}\right], \quad h_{2}=\left[\begin{array}{c}
-14.0897 \\
-9.0480
\end{array}\right] \text {. }
$$

By (46), the gain matrices $K_{i}, i=1,2$, can be obtained

$$
K_{1}=\left[\begin{array}{ll}
-1.5719 & -0.9805
\end{array}\right], K_{2}=\left[\begin{array}{ll}
-1.5502 & -0.9955
\end{array}\right] \text {. }
$$

Furthermore, from (47)-(48), we have $\mu_{1}=0.7499$, $\mu_{2}=2.2913, \mu=1.9739$ and $\tau_{a}^{*}=4.8790$. Choosing $\tau_{a}=5$, the simulation results are shown in Figs. 3-4, where the initial conditions are $x(0)=\left[\begin{array}{ll}0.6 & 0.4\end{array}\right]^{T}$ and $x(k)=\left[\begin{array}{ll}0 & 0\end{array}\right]^{T}, k=-2,-1$.

From Figs. 3-4, it is easy to see that the designed controller can guarantee that the resulting closed-loop system is positive and exponentially stable. This demonstrates the effectiveness of the proposed method.

\section{CONCLUSIONS}

The stabilization problems for both continuous-time and discrete-time positive switched systems with timevarying delays under asynchronous switching have been investigated in this paper. Sufficient conditions for the existence of the asynchronous controllers are established, and the desired controller gain matrices are obtained easily through the solutions of LMIs. Our future work will focus on studying the stability and stabilization problems for positive switched systems by using the mode-dependent average dwell time method proposed in [41].

\section{REFERENCES}

[1] E. R. Carson, C. Cobelli, and L. Finkelstein, "Modeling and identification of metabolic systems," American Journal of Physiology, vol. 240, no. 3, pp. R120-R129, 1981.

[2] H. Caswell, Matrix Population Models: Construction, Analysis and Interpretation, Sinauer Assoc., Sunderland, MA, 2001.

[3] L. Caccetta, L. R. Foulds, and V. G. Rumchev, "A positive linear discrete-time model of capacity planning and its controllability properties," Mathematical and Computer Modeling, vol. 40, no. 1-2, pp. 217-226, July 2004.

[4] R. Shorten, F. Wirth, and D. Leith, "A positive systems model of TCP-like congestion control: Asymptotic results," IEEE/ACM Trans. on Networking, vol. 14, no. 3, pp. 616-629, June 2006.

[5] R. Shorten, D. Leith, J. Foy, and R. Kilduff, "Towards an analysis and design framework for congestion control in communication networks," Proc. of the 12th Yale Workshop Adaptive and Learning Systems, 2003.

[6] A. Jadbabaie, J. Lin, and A. S. Morse, "Coordination of groups of mobile autonomous agents using nearest neighbor rules," IEEE Trans. on Automatic Control, vol. 48, no. 6, pp. 988-1001, June 2003.

[7] T. Kaczorek, "The choice of the forms of Lyapunov functions for a positive 2D Roesser model," International Journal of Applied Mathematics and 
Computer Science, vol. 17, no. 4, pp. 471-475, December 2007.

[8] X. Chen, J. Lam, P. Li, and Z. Shu, " $l_{1}$-induced norm and controller synthesis of positive systems," Automatica, vol. 49, no. 5, pp. 1377-1385, May 2013.

[9] M. Rami, F. Tadeo, and A. Benzaouia, "Control of constrained positive discrete systems," Proc. of the American Control Conference, New York, USA, pp. 5851-5856, July 2007,.

[10] M. Margaliot and M. S. Branicky, "Nice reachability for planar bilinear control systems with applications to planar linear switched systems," IEEE Trans. on Automatic Control, vol. 54, no. 6, pp. 1430-1435, June 2009.

[11] W. Xiang and J. Xiao, "stability analysis and control synthesis of switched impulsive systems," International Journal of Robust and Nonlinear Control, vol. 22, no. 13, pp. 1440-1459, September 2012.

[12] W. Xiang, J. Xiao, and M. N. Iqbal, "Asymptotic stability, $l_{2}$ gain boundness analysis and control synthesis for switched systems: a switching frequency approach," International Journal of Adaptive Control and Signal Processing, vol. 26, no. 4, pp. 350-373, April 2012.

[13] H. R. Karimi and H. Gao, "New delay-dependent exponential $H_{\infty}$ synchronization for uncertain neural networks with mixed time delays," IEEE Trans. on Systems, Man and Cybernetics Part B: Cybernetics, vol. 40, no. 1, pp. 173-185, February 2010.

[14] M. S. Mahmoud and P. Shi, "Robust stability, stabilization and $H_{\infty}$ control of time-delay systems with Markovian jump parameters," International Journal of Robust and Nonlinear Control, vol. 13, no.8, pp. 755-784, July 2003.

[15] Z. Xiang and R. Wang, "Robust control for uncertain switched non-linear systems with time delay under asynchronous switching," IET Control Theory and Applications, vol. 3, no. 8, pp. 10411050, August 2009.

[16] X. Zhao, L. Zhang, and P. Shi, "Stability of a class of switched positive linear time-delay systems," International Journal of Robust and Nonlinear Control, vol. 23, no. 5, pp. 578-589, March 2013.

[17] J. S. Zhang, Y. W. Wang, J. W. Xiao, and Y. J. Shen, "stability analysis of switched positive linear systems with stable and unstable subsystems," International Journal of Systems Science, DOI: 10.1080/002077 21.2013.770938, February 2013.

[18] J. Zhang and Z. Hang, "Robust stabilization of switched positive linear systems with uncertainties," International Journal of Control, Automation, and Systems, vol. 11, no. 1, pp. 41-47, February 2013.

[19] J. Zhang, Z. Han, F. Zhu, and J. Huang, "Stability and stabilization of positive switched systems with mode-dependent average dwell time," Nonlinear Analysis: Hybrid Systems, vol. 9, no. 1, pp. 42-55,
August 2013.

[20] X. Liu and C. Dang, "Stability analysis of positive switched linear systems with delays," IEEE Trans. on Automatic Control, vol. 56, no. 7, pp. 16841690, July 2011.

[21] Y. Zheng and G. Feng, "Stabilization of second order LTI switched positive systems," International Journal of Control, vol. 84, no. 8, pp. 1387-1397, August 2011.

[22] Y. Zheng and G. Feng, "Two slow stabilizing switching laws for discrete time positive switched systems," International Journal of Robust and Nonlinear Control, DOI: 10.1002/rnc. 3032, June 2013.

[23] E. Fornasini and M. Valcher, "Stability and stabilizability of special classes of discrete-time positive switched systems," Proc. of the American Control Conference, San Francisco, USA, pp. 2619-2624, July 2011.

[24] F. Najson, "State-Feedback stabilizability, optimality, and convexity in switched positive linear systems," Proc. of the American Control Conference, San Francisco, USA, pp. 2625-2632, July 2011.

[25] X. Zhao, L. Zhang, P. Shi, and M. Liu, "Stability of switched positive linear systems with average dwell time switching," Automatica, vol. 48, no. 6, pp. 1132-1137, June 2012.

[26] G. Xie and L. Wang, "Stabilization of switched linear systems with time-delay in detection of switching signal," Journal of Mathematical Analysis and Applications, vol. 305, no. 6, pp. 277290, May 2005.

[27] X. Zhao, P. Shi, and L. Zhang, "Asynchronously switched control of a class of slowly switched linear systems," Systems \& Control Letters, vol. 61, no. 12, pp. 1151-1156, December 2012.

[28] Z. Xiang, Y. N. Sun, and Q. Chen, "Robust reliable stabilization of uncertain switched neutral systems with delayed switching," Applied Mathematics and Computation, vol. 217, no. 23, pp. 9835-9844, August 2011.

[29] R. Wang, Z. G. Wu, and P. Shi, "Dynamic output feedback control for a class of switched delay systems under asynchronous switching," Information Sciences, vol. 225, pp. 72-80, March 2013.

[30] L. Zhang and P. Shi, "Stability, $l_{2}$-gain and asynchronous $H_{\infty}$ control of discrete-time switched systems with average dwell time," IEEE Trans. on Automatic Control, vol. 54, no. 9, pp. 2193-2200, September 2009.

[31] L. Zhang and H. Gao, "Asynchronously switched control of switched linear systems with average dwell time," Automatica, vol. 46, no. 5, pp. 953958, May 2010.

[32] W. Xiang, J. Xiao, and M. N. Iqbal, "Fault detection for switched nonlinear systems under asynchronous switching," International Journal of Control, vol. 84, no. 8, pp. 1362-1376, August 2011. 
[33] W. Xiang and J. Xiao, " $H_{\infty}$ filtering for switched nonlinear systems under asynchronous switching," International Journal of Systems Science, vol. 42, no. 5, pp. 751-765, May 2011.

[34] O. Mason and R. Shorten, "On linear copositive Lyapunov functions and the stability of switched positive linear systems," IEEE Trans. on Automatic Control, vol. 52, no. 7, pp. 1346-1349, July 2007.

[35] D. Wang, W. Wang, and P. Shi, "Exponential $H_{\infty}$ filtering for switched linear systems with interval time-varying delay," International Journal of Robust and Nonlinear Control, vol. 19, no. 5, pp. 532-551, March 2009.

[36] J. P. Hespanha and A. S. Morse, "Stability of switched systems with average dwell-time," Proc. of the 38th IEEE Conference on Decision and Control, Phoenix, AZ, vol. 3, pp. 2655-2660, December 1999.

[37] W. Zhang and L. Yu, "Stability analysis for discrete-time switched time-delay systems," Automatica, vol. 45, no. 10, pp. 2265-2271, October 2009.

[38] X. Liu, W. Yu, and L. Wang, "Stability analysis of positive systems with bounded time-varying delays," IEEE Trans. on Circuits and Systems II, vol. 56, no. 7, pp. 600-604, July 2009.

[39] M. Xiang and Z. Xiang, "Reliable $L_{1}$ control of positive switched systems with time-varying delays," Advances in Difference Equations, vol. 2013, no. 25, pp. 1-15, January 2013.

[40] M. Xiang and Z. Xiang, "Stability, $L_{1}$-gain and control synthesis for positive switched systems with time-varying delays," Nonlinear Analysis: Hybrid Systems, vol. 9, no. 1, pp. 9-17, August 2013.

[41] X. Zhao, L. Zhang, P. Shi, and M. Liu, "Stability and stabilization of switched linear systems with mode-dependent average dwell time," IEEE Trans. on Automatic Control, vol. 57, no. 7, pp. 18091815, July 2012. 